\title{
Voice analyses using smartphone-based data in patients with bipolar disorder, unaffected relatives and healthy control individuals, and during different affective states
}

Maria Faurholt-Jepsen ${ }^{1 *+} \mathbb{0}$, Darius Adam Rohani ${ }^{2+}$, Jonas Busk ${ }^{3}$, Maj Vinberg ${ }^{1,4}$, Jakob Eyvind Bardram ${ }^{2}$ and Lars Vedel Kessing ${ }^{1}$

\begin{abstract}
Background: Voice features have been suggested as objective markers of bipolar disorder (BD).

Aims: To investigate whether voice features from naturalistic phone calls could discriminate between (1) BD, unaffected first-degree relatives (UR) and healthy control individuals (HC); (2) affective states within BD.

Methods: Voice features were collected daily during naturalistic phone calls for up to 972 days. A total of 121 patients with BD, 21 UR and $38 \mathrm{HC}$ were included. A total of 107.033 voice data entries were collected [BD ( $\mathrm{n}$ $=78.733), \mathrm{UR}(\mathrm{n}=8004)$, and HC $(n=20.296)]$. Daily, patients evaluated symptoms using a smartphone-based system. Affective states were defined according to these evaluations. Data were analyzed using random forest machine learning algorithms.
\end{abstract}

Results: Compared to HC, BD was classified with a sensitivity of 0.79 (SD 0.11)/AUC $=0.76$ (SD 0.11) and UR with a sensitivity of 0.53 (SD 0.21)/AUC of 0.72 (SD 0.12). Within BD, compared to euthymia, mania was classified with a specificity of 0.75 (SD 0.16)/AUC $=0.66$ (SD 0.11). Compared to euthymia, depression was classified with a specificity of 0.70 (SD 0.16)/AUC $=0.66$ (SD 0.12). In all models the user dependent models outperformed the user independent models. Models combining increased mood, increased activity and insomnia compared to periods without performed best with a specificity of 0.78 (SD 0.16)/AUC $=0.67$ (SD 0.11).

Conclusions: Voice features from naturalistic phone calls may represent a supplementary objective marker discriminating $\mathrm{BD}$ from $\mathrm{HC}$ and a state marker within $\mathrm{BD}$.

Keywords: Voice analysis, Classification, Random Forest, Bipolar disorder, openSMILE

*Correspondence: maria@faurholt-jepsen.dk

${ }^{\dagger}$ Maria Faurholt-Jepsen and Darius Adam Rohani contributed equally to this work and are shared first authors

${ }^{1}$ Copenhagen Affective Disorder Research Center (CADIC), Psychiatric Center Copenhagen, Rigshospitalet, Blegdamsvej 9, 2100 Copenhagen, Denmark

Full list of author information is available at the end of the article

\section{Introduction}

Bipolar disorder (BD) is characterized by recurrent affective episodes with significant alterations in core features of mood, activity and sleep (Goodwin and Jamison 1996). There is a substantial diagnostic delay and a progression of illness severity during untreated years, stressing the need for earlier diagnosis and intervention (Baldessarini et al. 2007; Kessing et al. 2014). However, due to the lack of objective tests, the diagnostic process as well as the clinical assessment of illness activity relies on patient 
information, clinical evaluation and rating scales (Phillips and Kupfer 2013). This evaluation process involves a risk of individual observer bias and recall distortions at various levels (Silva et al. 2015; Silva et al. 2016). Therefore, objective supplementary methods for assisting clinicians in the diagnosis and the assessment of illness activity in $\mathrm{BD}$ would be a tremendous advantage.

It is well established that $20-30 \%$ of unaffected firstdegree relatives (UR) of patients with BD develop affective illness, compared to $2-5 \%$ among healthy control individuals (HC) (Vedel Kessing et al. 2021). Further, functioning is substantially and broadly decreased within education, employment, income, cohabitating and being married in UR compared with HC (Sletved et al. 2021). Therefore, it is likely that UR to patients with BD will show alterations in prodromal symptoms and features related to illness intermediate between patients with $\mathrm{BD}$ and $\mathrm{HC}$.

Speech is individual for each person like 'a fingerprint', and speech patterns have shown to provide indicators of mental disorders. In 1921, Emil Kraepelin emphasized that patients with depression tended to have lower pitch, lower speech rate and more monotonous speech (Lord et al. 1921), and studies analyzing the spoken language in affective disorders date back as early as 1938 (Newman and Mather 1938). Differences in language structure between patients with $\mathrm{BD}$ and $\mathrm{HC}$ have been described, and changes in speech has been suggested as objective, sensitive and valid measures of depressive and (hypo-) manic episodes in BD (Mundt et al. 2012; Raucher-Chéné et al. 2017; Arevian 2020). A recent systematic review concerning automated assessment of psychiatric disorders using speech suggested that speech processing technology could aid mental health assessments (Low et al. 2020). However, this review also addressed obstacles concerning the lack of larger, transdiagnostic and longitudinal studies (Low et al. 2020).

Digital phenotyping refers to approaches in which personal data gathered from mobile devices and sensors is analyzed to provide health information on physiological functions, or behavioral indicators, such as the user's speech (Insel 2017; Ebner-Priemer 2020). These data can be seen as digital footprints/digital markersor data traces arising as a by-product from interactions with technology. Software for ecologically extracting data on voice features from naturalistic phone calls has been developed (Eyben et al. 2010). Previous studies concerning voice features collected digitally within $\mathrm{BD}$ have investigated the use of speech to classify affective states and suggested that (hypo)manic states more accurately were classified than depressive states (Karam et al. 2014; Muaremi et al. 2014; Maxhuni et al. 2016; Gideon et al. 2016; Zhang et al. 2018; Vanello et al. 2012; Guidi et al.
2015). A previous study conducted by the authors found, that voice features collected in naturalistic settings using smartphones may represent objective state markers in patients with BD (Faurholt-Jepsen 2016). However, this previous study included a small number of patients and thus hold less strength. Moreover, it has not been investigated whether voice features collected from naturalistic phone calls can discriminate between BD, UR and HC. Smartphone-based voice technology could potentially aid clinicians in early diagnosing of BD and in identifying and targeting prodromal symptoms and states in UR.

\section{Objectives}

The present study aimed to investigate whether voice features collected from naturalistic phone calls (1) could discriminate between patients with $\mathrm{BD}, \mathrm{UR}$, and $\mathrm{HC}$; (2) within patients with BD could discriminate between (a) mania and euthymia and (b) depression and euthymia; and (3) within patients with BD could discriminate between (a) periods with increased activity and neutral activity, (b) periods with decreased activity and neutral activity, (c) periods with insomnia and periods without, and (d) periods with combined increased mood, increased activity and insomnia and periods without. We hypothesized that voice features would be able to discriminate between patients with $\mathrm{BD}$ and $\mathrm{HC}$, and between UR and $\mathrm{HC}$ (with UR intermediate between patients with $\mathrm{BD}$ and $\mathrm{HC}$ ), and further discriminate between states within patients with $\mathrm{BD}$.

\section{Methods and materials}

\section{Study design and participants}

The present study included data from two studies-the RADMIS trial (Faurholt-Jepsen et al. 2020) and the larger ongoing Bipolar Illness Onset study (BIO study) (Kessing 2017). Data were collected during the period from 2017 to 2020. All participants underwent The Schedules of Clinical Assessment in Neuropsychiatry (SCAN) interview (Wing et al. 1990) to confirm the clinical diagnosis of (or the lack of) BD.

\section{The RADMIS trial}

Patients with a diagnosis of BD who were hospitalized due to an affective episode and being discharged from one of five psychiatric centers at the Mental Health Services, Capital Region of Denmark, Denmark in the period from May 2017 to August 2019 were invited to participate in the RADMIS trial. Inclusion criteria: age above 18 years, BD diagnosis (ICD-10), discharge from a psychiatric hospital in The Capital Region of Denmark following an affective episode (depression, mania or mixed episode). Exclusion criteria: pregnancy and a lack of Danish language skills. In addition to standard treatment, 
patients were randomized with a balanced allocation ratio to either (1) daily use of a smartphone-based monitoring system (the Monsenso system-se description below) (the intervention group) or to (2) normal use of smartphones (the control group) during a 6 months follow-up period. Only patients from the intervention group providing smartphone-based data were included in the present study.

\section{The BIO study}

Three groups of participants were included in the BIO study: patients with newly diagnosed BD, UR, and HC.

\section{Patients with $B D$}

Inclusion criteria: a newly diagnosis of a single manic episode or BD (ICD-10) and ages between 15 and 70 years.

\section{UR}

UR, siblings or children, to the patients included in the BIO study, were recruited after permission from patients. Exclusion criteria: any previous or current psychiatric diagnosis lower than F34.0 (CD-10) (i.e., organic mental disorders, mental and behavioral disorders due to psychoactive substance use including alcohol, schizophrenia or other psychotic disorders, affective disorders).

\section{$H C$}

$\mathrm{HC}$ were recruited among blood donors, aged between 15 and 70 years, from the Blood Bank at Rigshospitalet, Copenhagen. Exclusion criteria: treatment requiring psychiatric disorder in the individual or one of the individuals' first-degree family members. All participants in the BIO study were offered to use a smartphone-based monitoring system on a daily basis (the Monsenso systemsee description below) during the study period.

\section{Clinical assessments}

Clinical evaluations of the severity of depressive and manic symptoms were conducted by a trained researcher using the Hamilton Depression Rating Scale 17-items (HDRS) (Hamilton 1967) and the Young Mania Rating Scale (YMRS) (Young et al. 1978).

\section{Patient-reported smartphone-based data}

A smartphone-based monitoring system (the Monsenso system) was installed on the participants own smartphones (both iPhone and Android smartphones). The smartphone-based monitoring system developed by the authors was used by the patients with $\mathrm{BD}$ on a daily basis to collect fine-grained real-time recordings of mood, activity, and sleep duration (Bardram et al. 2013). Mood was evaluated with scores on a 9-point scale ranging from depressed to manic $(-3,-2,-1,-0.5$,
$0,0.5,1,2,3)$. Euthymia mood was defined a priori as a mood score of $-0.5,0,0.5$. Depression was defined as mood score $<-0.5$, and mania was defined as mood score $>0.5$. Daily activity levels were rated on a 7-point scale $(-3,-2,-1,0,1,2,3)$ with 0 representing normal activity level. Sleep duration was calculated based on daily reports of bedtime and wake-up time. Insomnia was defined as total sleep duration $<360 \mathrm{~min}$. In addition, a broader definition of mania was made by combining increased mood $(>0.5)$, increased activity $(>0)$ and decreased sleep $(<360 \mathrm{~min}$.).

\section{Voice features}

Voice features were collected from the participants' phone calls (only Android smartphones) during their everyday life using the open-source Speech and Music Interpretation by Large-space Extraction (openSMILE vs. 2.1.0, Emo-Large) toolkit (Eyben et al. 2010; Schuller et al. 2010). The toolkit is a feature extractor for signal processing and machine learning applications, and it is designed for real-time processing. The toolkit used a built-in voice activity detection to live record voice samples from each incoming and outgoing phone call on the participants' smartphone. The voice activity detection was run solely on the study participants' onboard microphone such that the voice segments represented one recorded audio stream from the participant's voice. The audio stream was used to extract acoustic features 'online', e.g., directly on the study participants' smartphones for each phone call. Voice samples were deleted locally on the smartphone after each phone call, and thus there was not access to any content related material from phone calls. The EmoLarge was a predefined set consisting of 6552 features, e.g., pitch, loudness, and energy, represented through various 1st level descriptive statistics including means, regression coefficients, and percentiles. The set has been found to be particularly relevant for classifying emotions (Pfister and Robinson 2010).

\section{Statistical methods}

Data were imported to and processed in Python (version 3.8) with packages sklearn (v. 0.23.2), imblearn (v. 0.7.0), and pandas (v. 1.1.4).

Aim 1 concerned the discrimination between patients with $\mathrm{BD}, \mathrm{UR}$, and $\mathrm{HC}$ based on the use of collected voice features. Aims 2 and 3 concerned the use of voice data from patients with $\mathrm{BD}$ to classify the symptom class labels within mood, energy, and sleep collected daily from smartphones, and a combination of the three.

For all analyses Random Forest (RF) classifiers were built to discriminate between classes (Breiman 2001). The RF classifiers combine several decision tree classifiers into a single classifier. A RF model uses the 
ensemble technique to yield a prediction from multiple independent decision tree classifiers. RF models were chosen as they generally can handle large number of features while being robust to overfitting. Each tree is generated from a subsample of the data and using a random subset of features to ensure maximal degree of independence among the trees. The classifier uses supervised learning, i.e., information of the group status/affective state, to build nodes that split the dataset into groups. These splits continue until the model either has a group with only a single class, or if further splits are unable to improve the classification. Call entries with missing voice feature values and features with identical values (i.e., zero variance) were removed.

All classifications were binary (e.g., patients with $B D$ versus $H C$ ). For aim 2 and 3 patient-reported smartphone-based data for any specific day during the study period were included in the analyses if both voice features and patient-reported smartphone-based on mood, activity or sleep were available for the same day. We evaluated RF models on the resulting data set through a five-fold participant-based cross-validation. Five-fold cross-validation partitions the data in 5 parts of approximately the same size. Five to one partitions of the data were used to train the model, while the last partition was used to test the model, thereby evaluating the performance on unseen data samples. This was repeated 5 times so all samples were used for testing once to yield an average performance across all folds. We used a participant-based cross-validation version, where the test partition included participants that were not part of the training partition and vice versa. The participant-based method is particularly important for aim 1 since all voice data for each participant is identically labeled (i.e., either BD, UR, or HC). If the same participant is represented in the training and test partition the model would falsely learn to discriminate participant-based characteristics instead of clinical diagnose or state. Ad-hoc analyses without the participant-based cross-validation displayed significant better results. Therefore, to avoid learning on participant traits, all analyses included participant-based cross-validation.

In each cross-validation fold, the training set was used to calculate standardization parameters that transform the voice features training set to zero mean, unit variance. The calculated parameters were then applied to the test set. We used this standardization approach to create an unbiased data transformation invariant for factors such as gender, age, or microphone types selected by the phone vendors. As we used a participant-based cross-validation approach, the standardization was done for each voice feature across all participants.
Analyses concerning aims 2 and 3 were separated in two model types. First, a user-independent model thatas for aim 1-combines data from all participants in the same model. The model uses information from known participants to classify symptoms of unknown patients. Second, a user-dependent model personalized model for each patient was built.

We observed significant class imbalance in the data for all aims (e.g., fewer cases of symptoms of 'mania' compared with 'euthymia'). Therefore, we applied a resampling process on the training data to balance the two classes. We did a combination with SMOTE oversampling (Chawla et al. 2002) of the minority class to represent $33 \%$ of the cases, followed by random under sampling of the majority class until the sample size was identical to the minority class. The combination of oversampling with SMOTE and under sampling has previously been shown effective to counter class imbalance (García et al. 2016). Without a resampling scheme, the RF classifier would favor overrepresented classes. However, resampling was only performed on the training data, to keep the test set class distribution representative for the collected data. In the cases where class distribution was less than $33 \%$ skewed, we only performed random under sampling.

\section{Classifier performance}

We applied several standard metrics for binary classification computed on a test set held out data and compared the results to a majority vote baseline model.

The metrics included a) 'accuracy' (defined as the number of correct classifications of the positive and negative cases divided by the total number of cases); (b) 'F1-score' (estimates the model's ability to identify the positive class correctly, and was defined as the true positives divided by the true positives and the average between false positives and false negatives); (c) 'sensitivity' (defined as true positives divided by positives); (d) 'specificity' (defined as true negatives over all negatives); (e) 'area under the characteristic curve' (AUC) which is the area under the entire Receiver Operating Characteristic (ROC) curve. A ROC curve displays the model performance of sensitivity and specificity at all probability thresholds. The sensitivity and specificity reported in the tables are based on a threshold of $50 \%$. An AUC value of 0.5 represents random guessing, while a value of one is a perfect classifier. To further strengthen performance interpretation a Bayesian inference framework with intrinsic priors was added (B10) (Leon-Novelo et al. 2012). The method handles unbalanced data well as proven through various simulated and real work examples (Olivetti et al. 2015). The measure is based on a statistical foundation through a test of statistical independence between, here, our predicted results 
and the actual symptom registered. Therefore, a direct standardized guideline exists. A value below 0 indicates a negative evidence for a statistically dependency, a value between 1 and 3 suggests a more positive indication, 3-5 a strong indication, while a value above 5 is a decisive indication of statistically dependence.

All classification metrics were computed within each cross-validation fold to yield a mean (M) and standard deviation (SD) value across all five-folds. In the personalized model we further averaged across all patients.

For aim 1, we ran a randomized permutation model (Berry et al. 2002) to test whether voice data from the three populations were statistically significantly different from each other. We randomly shuffled the class label for each participant and re-ran the entire RF classification. This was repeated 200 times to generate a non-parametric null-distribution of AUC scores (Fig. 1). Statistically significance was determined if the RF test AUC statistics with true class labels exceeds the null distribution with a significance level of $\mathrm{p}=0.05$.

For aims 2 and 3, we developed a majority vote model and a random classifier as a baseline. Unlike the RF model, the baseline models did not include voice data. Simply, in the majority vote model, the most frequently observed class label in the training data, was used to classify test data. In cases where there was an equal class distribution, the test data was classified at random. The random classifier used a uniform distribution to randomly choose a class label.

\section{Ethical considerations The RADMIS trial}

The RADMIS trial was approved by the Regional Ethics Committee in The Capital Region of Denmark and the data agency, Capital Region of Copenhagen (H-16046093, RHP-2017-005, I-Suite: 05365) and registered at ClinicalTrials.gov (NCT03033420).

\section{The BIO study}

The study protocol was approved by the Committee on Health Research Ethics of the Capital region of Denmark (protocol No. H-7-2014-007) and the Danish Data Protection Agency, Capital Region of Copenhagen (RHP-2015-023).

Both studies complied with the Declaration of Helsinki (Seoul, October 2008). All participants provided written informed consent. Data from smartphones were stored by Monsenso subject to a data management agreement between Monsenso and The Capital Region of Denmark.

\section{Results}

\section{Background characteristics}

Overall, a total of 254 participants were included in the present study. A total of 27 participants dropped out of the study (mainly due to not having the time to participate), and a total of 47 participants did not provide voice data. Thus, a total 180 participants were available and included in the present report (patients with $\mathrm{BD} \mathrm{n}=121$, UR $\mathrm{n}=21$, and $\mathrm{HC} \mathrm{n}=38$ ). The participants provided on average $157(\mathrm{SD}=174)$ days

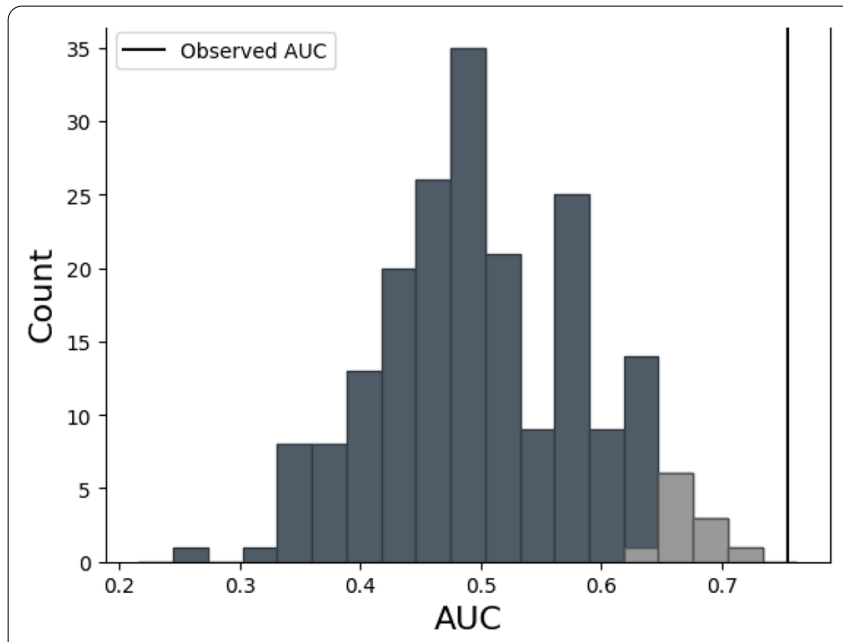

A

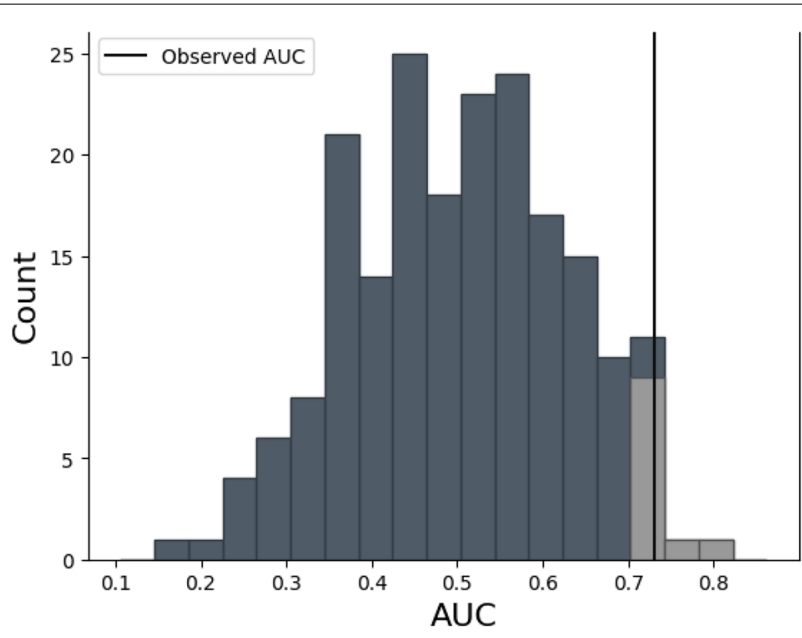

B

Fig. 1 A generated null distribution of AUC values from a permutation test where the class labels (e.g., patients with bipolar disorder and healthy controls) are randomly shuffled 200 times and an AUC value for each permutation is plotted. The light grey region represents the critical area with the $5 \%$ largest values. The vertical lines represent the observed AUC values from the true class labels. A Generated null-distribution for the Random Forest classification of patients with bipolar disorder against healthy control individuals. B Generated null-distribution for the Random Forest classification of patients with bipolar disorder against unaffected relatives 
with at least one voice recording with a range between 1 and 972 days.

A total of 107.033 voice data entries were collected across patients with BD ( $\mathrm{n}=78.733)$, UR $(\mathrm{n}=8004)$, and HC $(\mathrm{n}=20.296)$.

Overall, the participants had a mean age of 34.5 (SD $11.5)$ years with a range from 18 to 67 years. A total of $56 \%(\mathrm{n}=101)$ were women.

A total of $41 \%(n=49)$ of patients with BD had a HDRS score $\geq 13$ at inclusion, and a mean score on the YMRS of 3.76 (SD 4.71). Only $5 \%(n=6)$ of the patients had an YMRS score $\geq 13$ at inclusion. There were no statistically significant differences in age or sex distribution across the three populations. There was a statistically significant difference in education level (BD: $\mathrm{HC}, \mathrm{p}=0.03$ ) and unemployment (BD: $\mathrm{HC}$, $\mathrm{p}=0.001$; $\mathrm{BD}$ : UR, $\mathrm{p}=0.021)$ between patients with $\mathrm{BD}, \mathrm{HC}$ and UR. Further background characteristics are presented in Table 1.

\section{Classification of groups}

Table 2 present the results for classification of patients with BD (78.731 observations), HC (20.296 observations) and UR (8004 observations) based on voice features.

The sensitivity and specificity for classifying patients with $\mathrm{BD}$ versus $\mathrm{HC}$ was 0.79 (SD 0.11) and 0.54 (SD 0.20), respectively and with an AUC of 0.76 (SD 0.11). The sensitivity and specificity for classifying patients with $\mathrm{BD}$ versus UR was 0.73 (SD 0.07) and 0.28 (SD 0.11), respectively with an AUC of 0.52 (SD 0.09). The sensitivity and specificity for classifying UR versus $\mathrm{HC}$ was 0.53 (SD 0.21 ) and 0.67 (SD 0.24), respectively and with an AUC of 0.72 (SD 0.12). Figure 1A, B presents the generated nulldistribution of AUC scores from permuted class labels as generated from the randomized permutation model. The lighter area shows the critical level for a one-tail test with a significance level of 0.05 , e.g., values that are high enough to be considered statistically significant at

Table 1 Background characteristics of participants, $n=180$

\begin{tabular}{|c|c|c|c|c|}
\hline & $\begin{array}{l}\text { Patients with bipolar } \\
\text { disorder (BD) }\end{array}$ & $\begin{array}{l}\text { Healthy control } \\
\text { individuals }(\mathrm{HC})\end{array}$ & $\begin{array}{l}\text { Unaffected relatives } \\
\text { (UR) }\end{array}$ & $\mathrm{p}$ \\
\hline $\mathrm{n}, \%$ female & $121(60.0)$ & $38(45.0)$ & $21(52.0)$ & $p>0.16$ \\
\hline Age & $35.71(12.35)$ & $31.66(10.87)$ & $32.29(10.57)$ & $p>0.16$ \\
\hline Employed, \% (n) & $17(20)$ & $50(19)$ & $57(12)$ & $\begin{array}{l}\text { BD:HC ( } p=0.002) \\
\text { UR:HC ( } p=0.88)\end{array}$ \\
\hline Student, \% (n) & $31(37)$ & $39(15)$ & $33(7)$ & $p>0.42$ \\
\hline Unemployed, \% (n) & $30(36)$ & $8(3)$ & $10(2)$ & $\begin{array}{l}\text { BD:HC }(p=0.001) \\
\text { UR:HC }(p=0.76)\end{array}$ \\
\hline Education (years) & $13.68(4.66)$ & $15.58(1.57)$ & $15.07(2.62)$ & $\begin{array}{l}\text { BD:HC ( } p=0.03) \\
\text { UR:HC ( } p=0.89)\end{array}$ \\
\hline Bipolar subtype I, \% (n) & $38(46)$ & N/A & N/A & N/A \\
\hline HAMD at inclusion & $10.84(6.93)$ & $0.95(1.62)$ & $3.10(3.32)$ & $\begin{array}{l}\text { BD:HC }(p<0.001) \\
\text { UR:HC }(p=0.38)\end{array}$ \\
\hline YMRS at inclusion & $3.76(4.71)$ & $0.51(0.98)$ & $1.25(1.92)$ & $\begin{array}{l}\text { BD:HC }(p<0.001) \\
\text { UR:HC }(p=0.77)\end{array}$ \\
\hline Previous hospitalizations (number) & $4[1-50]$ & $\mathrm{N} / \mathrm{A}$ & N/A & N/A \\
\hline Previous depressive episodes (number) & $10[1-80]$ & N/A & N/A & N/A \\
\hline Previous (hypo)manic episodes (number) & $14[1-182]$ & N/A & N/A & N/A \\
\hline IIIness duration (years) & $14.86(10.37)$ & $\mathrm{N} / \mathrm{A}$ & $\mathrm{N} / \mathrm{A}$ & N/A \\
\hline \multicolumn{5}{|l|}{ Psychotropic medication } \\
\hline Anticonvulsant, \% (n) & $42(51)$ & N/A & N/A & N/A \\
\hline Lithium, \% (n) & $48(58)$ & N/A & N/A & N/A \\
\hline Antipsychotics, \% (n) & $49(59)$ & N/A & N/A & N/A \\
\hline Antidepressants, \% (n) & $18(22)$ & $\mathrm{N} / \mathrm{A}$ & $\mathrm{N} / \mathrm{A}$ & $\mathrm{N} / \mathrm{A}$ \\
\hline
\end{tabular}

Data are mean (SD), median [IQR] or proportions $(\%, \mathrm{n})$ unless otherwise stated HAMD Hamilton Depression Rating Scale 17-items score YMRS Young Mania Rating Scale score 
Table 2 Discrimination between patients with bipolar disorder (BD) $(n=121)$, unaffected relatives (UR) $(n=21)$ and healthy control individuals $(H C)(n=38)$ based on voice features collected from smartphones, $n=180$

\begin{tabular}{|c|c|c|c|c|c|c|}
\hline $\begin{array}{l}\text { Binary classifier ( } n=\text { number of } \\
\text { observations) }\end{array}$ & Model type & Accuracy (SD) & F1 score (SD) & Sensitivity (SD) & Specificity (SD) & $A U C(S D)$ \\
\hline \multirow{2}{*}{$\begin{array}{l}\mathrm{BD}(\mathrm{n}=78,731) \text { compared with HC (n} \\
=20,296)\end{array}$} & Random Forest model & $0.72(0.09)$ & $0.81(0.07)$ & $0.79(0.11)$ & $0.54(0.20)$ & $0.76(0.11)$ \\
\hline & Majority vote & $0.67(0.00)$ & $0.88(0.06)$ & $1.0(0.00)$ & $0.00(0.00)$ & $0.50(0.00)$ \\
\hline \multirow{2}{*}{$\begin{array}{l}\mathrm{BD}(\mathrm{n}=78,731) \text { compared with UR ( } \mathrm{n} \\
=8004)\end{array}$} & Random Forest model & $0.68(0.049)$ & $0.81(0.03)$ & $0.73(0.07)$ & $0.28(0.11)$ & $0.52(0.09)$ \\
\hline & Majority vote & $0.95(0.00)$ & $0.95(0.02)$ & $1.00(0.00)$ & $0.00(0.00)$ & $0.50(0.00)$ \\
\hline \multirow{2}{*}{$\begin{array}{l}\text { UR }(n=8004) \text { compared with HC ( } n \\
=20,296)\end{array}$} & Random Forest model & $0.59(0.13)$ & $0.38(0.15)$ & $0.53(0.21)$ & $0.67(0.24)$ & $0.72(0.12)$ \\
\hline & Majority vote & $0.80(0.00)$ & $0.00(0.00)$ & $0.00(0.00)$ & $1.00(0.00)$ & $0.50(0.00)$ \\
\hline
\end{tabular}

the 0.05 level. The horizontal line represent the observed AUC value from Table 2 for the correct class labels. In both cases, the observed AUC for patients with BD versus $\mathrm{HC}(0 / 200, \mathrm{p}<0.001)$ and UR versus $\mathrm{HC}(6 / 200$, $\mathrm{p}=0.03)$ differed statistically significantly. Thus, there was a statistically significant difference in voice feature between patients with $\mathrm{BD}$ versus $\mathrm{HC}$ and a statistically significant difference in voice features between UR and $\mathrm{HC}$.

\section{Classifications of states within bipolar disorder}

A total of 100 patients with BD provided both voice features and smartphone-based patient-reported data. Table 3 present the results for classification of different states in patients with $\mathrm{BD}$. In all the models presented in Table 3, the personalized user-dependent models outperformed the general user-independent models. Therefore, the results from the user-dependent models are presented below.

\section{Mania (1205 observations) versus euthymia (38.329 observations)}

The sensitivity and specificity for classifying mania versus euthymia was 0.42 (SD 0.22) and 0.75 (SD 0.16), respectively and with an AUC of 0.66 (SD 0.11).

\section{Depression (5329 observations) versus euthymia}

The sensitivity and specificity for classifying depression versus euthymia was 0.53 (SD 0.22) and 0.70 (SD 0.16), respectively and with an AUC of 0.66 (SD 0.12).

\section{Increased activity (12.890 observations) versus neutral activity (21.661 observations)}

The sensitivity and specificity for classifying increased activity versus neutral activity was 0.55 (SD 0.26) and 0.58 (SD 0.24), respectively and with an AUC of 0.61 (SD $0.10)$.

\section{Decreased activity (10.228 observations) versus neutral activity}

The sensitivity and specificity for classifying decreased activity versus neutral activity was 0.53 (SD 0.20) and 0.65 (SD 0.21), respectively and with an AUC of 0.62 (SD $0.10)$.

\section{Insomnia (8474 observations) versus periods without (36.140)}

The sensitivity and specificity for classifying insomnia versus periods without was 0.39 (SD 0.22) and 0.73 (SD 0.17 ), respectively and with an AUC of 0.59 (SD 0.08).

\section{Combined increased mood, increased activity and insomnia (471 observations) versus periods without (43.243 observations)}

The sensitivity and specificity for classifying combined increased mood, increased activity and insomnia versus periods without was 0.41 (SD 0.21) and 0.78 (SD 0.16), respectively and with an AUC of 0.67 (SD 0.11).

Figure 2 presents the association between patientreported mood and clinical ratings of depressive and manic symptoms according to the HDRS ( $\mathrm{r}=-0.64$, $\mathrm{p}$ $<0.001)$ and the YMRS $(\mathrm{r}=0.39, \mathrm{p}<0.001)$. In both cases the correlation coefficients were statistically significant.

The ROC curves generated by aggregating all model estimates and the corresponding true class labels in each cross-validation fold, as well as each patient in the userdependent classifiers, are presented in Fig. 3. As can be seen, the ROC curve for the sleep model is the closest to random, while the combined increased mood, increased activity and insomnia versus periods without performed best.

\section{Discussion}

The present study investigated the use of voice features collected during naturalistic phone calls for classifications of patients with $\mathrm{BD}, \mathrm{HC}$ and UR, as well as state classifications within patients with BD. First, and in accordance with our hypotheses, voice features rather sensitively discriminated $\mathrm{BD}$ from $\mathrm{HC}$, but in contrast 
Table 3 Classification within patients with bipolar disorder $(n=100)$ according to patient-reported smartphone-based data on mood, activity and sleep ${ }^{a}$

\begin{tabular}{|c|c|c|c|c|c|c|c|}
\hline $\begin{array}{l}\text { Binary classifier } \\
\mathrm{n}=\text { number of } \\
\text { observations }\end{array}$ & Model type & Accuracy (SD) & F1 score (SD) & Sensitivty (SD) & Specificity (SD) & AUC (SD) & $\mathrm{B} 10(\mathrm{SD})^{\mathrm{b}}$ \\
\hline \multicolumn{8}{|l|}{ Mood } \\
\hline \multirow[t]{6}{*}{$\begin{array}{l}\text { Mania }(n=1205) \text { versus } \\
\text { euthymia }(n=38,329)\end{array}$} & $\begin{array}{l}\text { Random Forest model- } \\
\text { user independent }\end{array}$ & $0.72(0.18)$ & $0.05(0.03)$ & $0.23(0.12)$ & $0.73(0.19)$ & $0.51(0.07)$ & $-2.65(0.34)$ \\
\hline & $\begin{array}{l}\text { Random Forest model- } \\
\text { user dependent }\end{array}$ & $0.74(0.16)$ & $0.25(0.22)$ & $0.42(0.22)$ & $0.75(0.16)$ & $0.66(0.11)$ & $0.53(3.69)$ \\
\hline & $\begin{array}{l}\text { Majority vote-user inde- } \\
\text { pendent }\end{array}$ & $0.94(0.00)$ & $0.00(0.00)$ & $0.00(0.00)$ & $1.00(0.00)$ & $0.50(0.00)$ & $-3.18(1.61)$ \\
\hline & $\begin{array}{l}\text { Majority vote-user } \\
\text { dependent }\end{array}$ & $0.97(0.00)$ & $0.08(0.25)$ & $0.11(0.31)$ & $0.89(0.31)$ & $0.48(0.02)$ & $-3.23(1.35)$ \\
\hline & $\begin{array}{l}\text { Random model-user } \\
\text { independent }\end{array}$ & $0.49(0.00)$ & $0.06(0.03)$ & $0.49(0.05)$ & $0.50(0.01)$ & $0.50(0.00)$ & $-2.82(0.27)$ \\
\hline & $\begin{array}{l}\text { Random model-user } \\
\text { dependent }\end{array}$ & $0.49(0.00)$ & $0.15(0.09)$ & $0.52(0.03)$ & $0.48(0.03)$ & $0.50(0.00)$ & $-2.21(0.59)$ \\
\hline \multirow{6}{*}{$\begin{array}{l}\text { Depression }(n=5329) \\
\text { versus euthymia }(\mathrm{n} \\
=38,329)\end{array}$} & $\begin{array}{l}\text { Random Forest model- } \\
\text { user independent }\end{array}$ & $0.63(0.06)$ & $0.21(0.07)$ & $0.40(0.07)$ & $0.66(0.05)$ & $0.55(0.05)$ & $-1.82(0.22)$ \\
\hline & $\begin{array}{l}\text { Random Forest model- } \\
\text { user dependent }\end{array}$ & $0.70(0.13)$ & $0.40(0.21)$ & $0.53(0.22)$ & $0.70(0.16)$ & $0.66(0.12)$ & $2.78(5.49)$ \\
\hline & $\begin{array}{l}\text { Majority vote-user inde- } \\
\text { pendent }\end{array}$ & $0.89(0.00)$ & $0.00(0.00)$ & $0.00(0.00)$ & $1.00(0.00)$ & $0.50(0.00)$ & $-3.78(1.31)$ \\
\hline & $\begin{array}{l}\text { Majority vote-user } \\
\text { dependent }\end{array}$ & $0.71(0.00)$ & $0.11(0.28)$ & $0.13(0.33)$ & $0.88(0.33)$ & $0.49(0.02)$ & $-3.29(0.00)$ \\
\hline & $\begin{array}{l}\text { Random model-user } \\
\text { independent }\end{array}$ & $0.50(0.00)$ & $0.24(0.06)$ & $0.51(0.01)$ & $0.49(0.00)$ & $0.50(0.00)$ & $-2.07(0.16)$ \\
\hline & $\begin{array}{l}\text { Random model-user } \\
\text { dependent }\end{array}$ & $0.46(0.0)$ & $0.31(0.17)$ & $0.55(0.07)$ & $0 . .44(0.05)$ & $0.50(0.00)$ & $-1.80(0.46)$ \\
\hline \multicolumn{8}{|l|}{ Activity } \\
\hline \multirow{6}{*}{$\begin{array}{l}\text { Increased }(n=12,890) \\
\text { versus neutral }(n \\
=21,661)\end{array}$} & $\begin{array}{l}\text { Random Forest model- } \\
\text { user independent }\end{array}$ & $0.46(0.08)$ & $0.43(0.10)$ & $0.55(0.07)$ & $0.41(0.09)$ & $0.48(0.07)$ & $-1.38(0.20)$ \\
\hline & $\begin{array}{l}\text { Random Forest model- } \\
\text { user dependent }\end{array}$ & $0.67(0.13)$ & $0.55(0.25)$ & $0.55(0.26)$ & $0.58(0.24)$ & $0.61(0.10)$ & $1.85(5.92)$ \\
\hline & $\begin{array}{l}\text { Majority vote-user inde- } \\
\text { pendent }\end{array}$ & $0.48(0.00)$ & $0.00(0.00)$ & $0.00(0.00)$ & $1.00(0.00)$ & $0.50(0.00)$ & $-3.52(0.00)$ \\
\hline & $\begin{array}{l}\text { Majority vote-user } \\
\text { dependent }\end{array}$ & $0.57(0.00)$ & $0.45(0.40)$ & $0.56(0.49)$ & $0.44(0.49)$ & $0.49(0.01)$ & $-0.02(0.15)$ \\
\hline & $\begin{array}{l}\text { Random model-user } \\
\text { independent }\end{array}$ & $0.50(0.00)$ & $0.45(0.07)$ & $0.50(0.01)$ & $0.49(0.01)$ & $0.50(0.00)$ & $-1.76(0.07)$ \\
\hline & $\begin{array}{l}\text { Random model-user } \\
\text { dependent }\end{array}$ & $0.53(0.00)$ & $0.50(0.13)$ & $0.56(0.05)$ & $0.45(0.05)$ & $0.50(0.00)$ & $-1.44(0.63)$ \\
\hline \multirow{6}{*}{$\begin{array}{l}\text { Decreased }(n=10,288) \\
\text { versus neutral }(n \\
=21,661)\end{array}$} & $\begin{array}{l}\text { Random Forest model- } \\
\text { user independent }\end{array}$ & $0.50(0.03)$ & $0.42(0.06)$ & $0.58(0.04)$ & $0.47(0.05)$ & $0.54(0.02)$ & $-1.43(0.42)$ \\
\hline & $\begin{array}{l}\text { Random Forest model- } \\
\text { user dependent }\end{array}$ & $0.66(0.11)$ & $0.53(0.18)$ & $0.53(0.20)$ & $0.65(0.21)$ & $0.62(0.10)$ & $3.13(6.40)$ \\
\hline & $\begin{array}{l}\text { Majority vote-user inde- } \\
\text { pendent }\end{array}$ & $0.69(0.00)$ & $0.00(0.00)$ & $0.00(0.00)$ & $1.00(0.00)$ & $0.50(0.00)$ & $-3.04(0.00)$ \\
\hline & $\begin{array}{l}\text { Majority vote-user } \\
\text { dependent }\end{array}$ & $0.71(0.00)$ & $0.27(0.37)$ & $0.35(0.47)$ & $0.65(0.47)$ & $0.49(0.02)$ & $-0.02(0.15)$ \\
\hline & $\begin{array}{l}\text { Random model-user } \\
\text { independent }\end{array}$ & $0.50(0.00)$ & $0.41(0.07)$ & $0.51(0.01)$ & $0.49(0.01)$ & $0.50(0.00)$ & $-1.77(0.10)$ \\
\hline & $\begin{array}{l}\text { Random modeluser } \\
\text { dependent }\end{array}$ & $0.55(0.00)$ & $0.45(0.12)$ & $0.55(0.05)$ & $0.44(0.05)$ & $0.50(0.00)$ & $-1.39(0.66)$ \\
\hline
\end{tabular}


Table 3 (continued)

\begin{tabular}{|c|c|c|c|c|c|c|c|}
\hline $\begin{array}{l}\text { Binary classifier } \\
n=\text { number of } \\
\text { observations }\end{array}$ & Model type & Accuracy (SD) & F1 score (SD) & Sensitivty (SD) & Specificity (SD) & AUC (SD) & $\mathrm{B} 10(\mathrm{SD})^{\mathrm{b}}$ \\
\hline \multicolumn{8}{|l|}{ Sleep } \\
\hline \multirow{6}{*}{$\begin{array}{l}\text { Insomnia }(n=8474) \\
\text { versus normal sleep } \\
(n=36,140)\end{array}$} & $\begin{array}{l}\text { Random Forest model- } \\
\text { user independent }\end{array}$ & $0.70(0.05)$ & $0.13(0.03)$ & $0.16(0.10)$ & $0.82(0.09)$ & $0.48(0.04)$ & $-1.49(0.43)$ \\
\hline & $\begin{array}{l}\text { Random Forest model- } \\
\text { user dependent }\end{array}$ & $0.70(0.16)$ & $0.33(0.18)$ & $0.39(0.22)$ & $0.73(0.17)$ & $0.59(0.08)$ & $0.28(2.66)$ \\
\hline & $\begin{array}{l}\text { Majority vote-user inde- } \\
\text { pendent }\end{array}$ & $0.85(0.00)$ & $0.00(0.00)$ & $0.00(0.00)$ & $1.00(0.00)$ & $0.50(0.00)$ & $-3.13(1.17)$ \\
\hline & $\begin{array}{l}\text { Majority vote-user } \\
\text { dependent }\end{array}$ & $0.90(0.00)$ & $0.05(0.17)$ & $0.06(0.24)$ & $0.93(0.24)$ & $0.49(0.01)$ & $-3.36(1.19)$ \\
\hline & $\begin{array}{l}\text { Random model-user } \\
\text { independent }\end{array}$ & $0.50(0.00)$ & $0.25(0.07)$ & $0.50(0.01)$ & $0.49(0.00)$ & $0.50(0.00)$ & $-2.03(0.13)$ \\
\hline & $\begin{array}{l}\text { Random model-user } \\
\text { dependent }\end{array}$ & $0.48(0.00)$ & $0.32(0.13)$ & $0.55(0.05)$ & $0.44(0.04)$ & $0.50(0.00)$ & $-1.77(0.48)$ \\
\hline \multicolumn{8}{|c|}{ A broader definition of mania } \\
\hline \multicolumn{8}{|c|}{ Combined increased mood, activity and decreased sleep } \\
\hline \multirow{6}{*}{$\begin{array}{l}\text { Combined increased } \\
\text { mood, activity and } \\
\text { decreased sleep } \\
(n=471) \text { versus rest } \\
(n=43,243)\end{array}$} & $\begin{array}{l}\text { Random Forest model- } \\
\text { user independent }\end{array}$ & $0.77(0.10)$ & $0.03(0.02)$ & $0.29(0.17)$ & $0.77(0.11)$ & $0.58(0.06)$ & $-1.68(0.28)$ \\
\hline & $\begin{array}{l}\text { Random Forest model- } \\
\text { user dependent }\end{array}$ & $0.77(0.15)$ & $0.17(0.16)$ & $0.41(0.21)$ & $0.78(0.16)$ & $0.67(0.11)$ & $2.15(6.02)$ \\
\hline & $\begin{array}{l}\text { Majority vote-user inde- } \\
\text { pendent }\end{array}$ & $0.98(0.00)$ & $0.00(0.00)$ & $0.00(0.00)$ & $1.00(0.00)$ & $0.50(0.00)$ & $-3.44(0.00)$ \\
\hline & $\begin{array}{l}\text { Majority vote-user } \\
\text { dependent }\end{array}$ & $0.99(0.00)$ & $0.00(0.00)$ & $0.00(0.00)$ & $1.00(0.00)$ & $0.48(0.02)$ & $-3.43(1.30)$ \\
\hline & $\begin{array}{l}\text { Random model-user } \\
\text { independent }\end{array}$ & $0.49(0.00)$ & $0.13(0.05)$ & $0.50(0.00)$ & $0.49(0.00)$ & $0.50(0.00)$ & $-2.39(0.18)$ \\
\hline & $\begin{array}{l}\text { Random model-user } \\
\text { dependent }\end{array}$ & $0.46(0.00)$ & $0.46(0.18)$ & $0.56(0.02)$ & $0.44(0.04)$ & $0.50(0.00)$ & $-1.78(0.24)$ \\
\hline
\end{tabular}

The number of observations is the recorded samples before any resampling or cross-validation partitioning

${ }^{a}$ Euthymia was defined as a mood score of $-0.5,0,0.5$. Depression was defined as a mood score $<-0.5$, and mania was defined as a mood score $>0.5$. Increased activity was defined as a score $>0$, and decreased activity was defined as a score $<0$. Insomnia was defined as total sleep duration $<360$ min. A broader definition of mania was made by combining increased mood, increased activity and decreased sleep

${ }^{\mathrm{b}}$ Bayesian inference framework
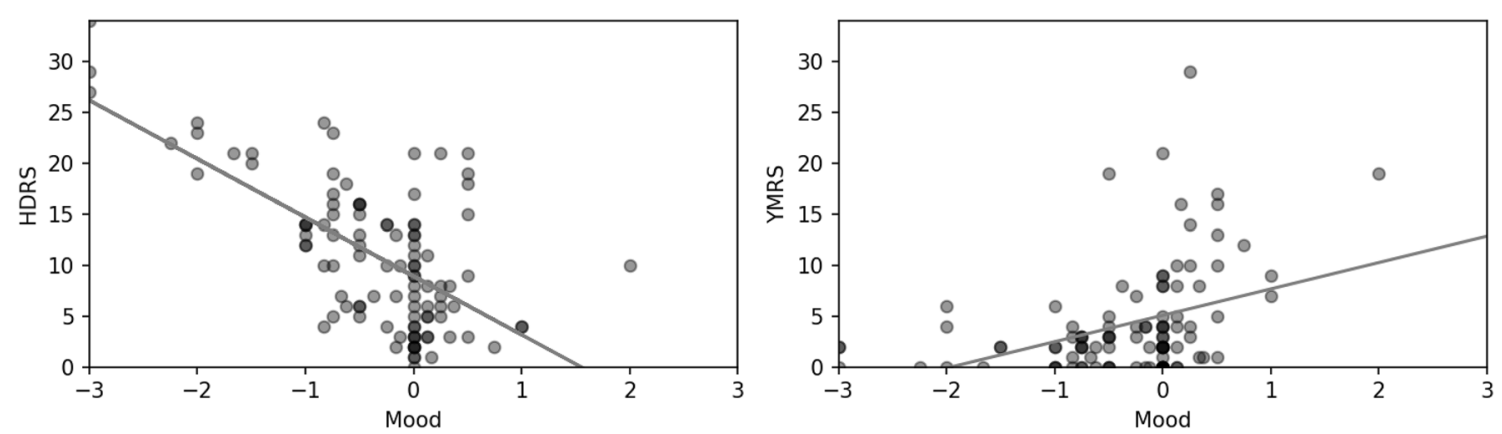

Fig. 2 The association between Hamilton Depression Rating Scale 17-items score (HDRS), Young Mania Rating Scale (YMRS) and patient-reported smartphone-based data on mood. The grey line indicates the linear least square fit for each combination

to our hypotheses with low specificity. Further, voice features statistically significantly differed between UR and $\mathrm{HC}$, but in contrast to our hypotheses discriminated between the two groups with a modest accuracy.
Second, within patients with BD, voice features during mania (and to a lesser degree during depression) rather specifically discriminated from voice features during euthymia, but with low sensitivity. In addition, voice 


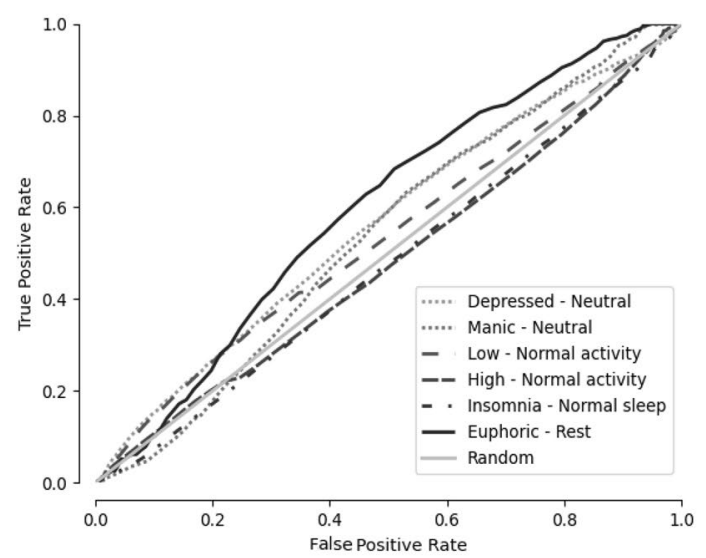

A

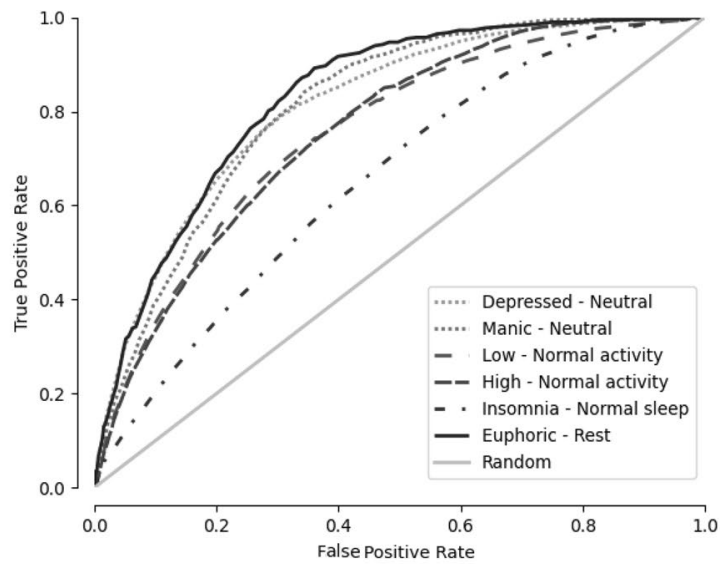

B

Fig. 3 The ROC curve for the classifications of different states based on voice features in patients with bipolar disorder. A The user-independent models; $\mathbf{B}$ the user-dependent models. Euphoric defined as combined increased mood and increased activity

features during periods with insomnia quite specifically discriminated from periods without insomnia. The model including voice features during periods with combined increased mood, increased activity and insomnia performed best among all the models as reflected by the specificity and the AUC. In line with our previous study (Faurholt-Jepsen 2016), within patients with BD the user dependent models clearly performed the best for classifications of different states, suggesting that changes in voice features is individual, like 'a fingerprint'.

Increased energy or activity has been highlighted in the DSM-5 and must now be present alongside mood changes to diagnose hypomania/mania raising energy/ activity to criterion A (Kessing et al. 2021; Fredskild et al. 2021). Nevertheless, in contrast to our hypotheses, the sensitivity and specificity for discriminating between increased activity and neutral activity, and decreased activity and neutral activity was low.

Interestingly, the findings from the present study are in line with findings from previous studies suggesting that voice features may be used as a trait (Zhang et al. 2018) and state (Karam et al. 2014; Gideon et al. 2016; Vanello et al. 2012; Guidi et al. 2015; Faurholt-Jepsen 2016) marker in BD. However, previous studies included rather small samples of patients with BD and did not compare voice features to UR and $H C$.

A recent systematic review concerning automated assessment of psychiatric disorders using speech suggested that speech processing technology could aid mental health assessments (Low et al. 2020). Many people own and use a smartphone and smartphones comprise a unique platform for unobtrusive and continuous monitoring. Due to the limited access to treatment facilities, during the past 10 years, and especially during the
COVID-19 pandemic, there has been an increase in the international interest in the use of mHealth technologies within mental health (Insel 2017; Wang et al. 2018; Anthes 2016; Patoz et al. 2021; Lagan 2020).

Within BD there is a substantial diagnostic delay, a progression of illness severity during untreated years, and a potential delayed intervention on subsyndromal symptoms. Assessments within BD are intermittent and may be limited partly due to the episodic nature of the disorder. The findings from the present study suggest that voice features may be used as an objective supplementary assessment method for diagnosis and identification of deterioration following initial informed consent. Such data has the additional advantage that they may be available when patients suffer from severe mood episodes and even when patients are non-adherent to treatment and don't attend clinical appointments.

\section{Advantages and limitations}

The present study was the first to include a large sample of both patients with $\mathrm{BD}, \mathrm{HC}$ and UR, and is therefore hypothesis generating within the field. Furthermore, the patients with $\mathrm{BD}$ were followed for a long time period allowing for collection of both fine-grained voice and daily patient-reported data. The affective states within patients with BD were defined according to daily patientreported smartphone-based data on mood, activity and sleep. In this way voice features and information on states was available for a larger proportion of days than if states were solely defined according to clinical ratings, which were conducted less frequently. While patient-reported smartphone-based mood was associated with scores on the HDRS and YMRS, a larger error margin was observed in the euthymic state ( -0.5 to 0.5$)$. The ability for the 
model to discriminate from a euthymic state might be affected by the patient's ability to self-assess when situated in the border between different states. Exploratory analyses investigating the classification of manic episodes using a cut-off on patient-reported smartphone-based mood $>1$ did not alter the estimates. Further, there may be a risk that the patient-reported smartphone-based data on mood were not missing at random, and thus voice feature during the most severe affective states might not have been included. The three included populations were well-characterized according to clinical as well as research-based assessments using the SCAN interview, and the patients with BD were newly diagnosed increasing the impact of the findings. A potential confounding effect of factors related to the mental health status of the included participants such as psychopharmacological treatment cannot be ruled out (Bock 2019). Future studies could consider investigating this aspect further.

In the present study, the sensitivity of discriminating between different affective states within BD was quite low, as reflected by the relatively modest AUCs. Considerations between the trade-off between the sensitivity and the specificity should be a priority in future studies.

The available voice features were collected unobtrusively during naturalistic settings reducing the Hawthorne effect (Wickström and Bendix 2000). The study included a large amount of fine-grained repeated data for each participant during long-term follow-up. However, in some of the analyses a low number of observations were included. The finding that within patients with $\mathrm{BD}$, the user dependent models outperformed the user independent models, and the user independent models performed close to random, suggest that change in voice features are highly individual, and thus hard to generalize between individuals.

The present study included the Speech and Music Interpretation by Large-space Extraction (openSMILE emolarge) feature set. It is possible that other configurations of the openSMILE toolkit or other feature extraction technologies, and subsequent features selections, to the one used in the present study could be feasible while keeping or improving the classification. This would help to reduce computational costs and save storage space. Further, from the present study, it was not possible to extract which of the included voice features that were most contributing to the classification models. The present study included patients with BD, but not patients with other psychiatric disorders. Future studies investigating the use of voice features for differentiating between psychiatric disorders could provide exciting information within the area. The voice features were extracted during regular phone calls, and thus we did not have access to voice feature from communication using other smartphone-based platforms.

\section{Perspectives and future implications}

Using voice features reflects a potential innovative, objective and unobtrusive supplementary method for discriminating patients with $\mathrm{BD}$ and UR from $\mathrm{HC}$ and as a state marker within patients with $\mathrm{BD}$.

\section{Conclusions}

The present study investigated for the first time the use of voice features collected during naturalistic phone calls in a large sample of patients with BD, HC and UR and for state classifications within $\mathrm{BD}$. It was shown that voice features can discriminate BD from $\mathrm{HC}$ with high sensitivity, but with low specificity, and that voice features significantly can differentiate between UR and HC. Within patients with $\mathrm{BD}$, mania was rather specifically discriminated from euthymia. However, the trade-off between the sensitivity and the specificity was in all models reflected by the modest AUCs.

Within patients with BD the user dependent models clearly performed the best for classifications of different states, suggesting that changes in voice features is individual, like 'a fingerprint'. These results show that voice features collected during naturalistic phone calls could potentially be used as a supplementary objective marker discriminating patients with $\mathrm{BD}$ from $\mathrm{HC}$ and as a state marker within patients with $\mathrm{BD}$.

\section{Abbreviations \\ BD: Bipolar disorder; UR: Unaffected first-degree relatives; HC: Healthy control individuals; SCAN: The Schedules of Clinical Assessment in Neuropsychiatry interview; HDRS: Hamilton Depression Rating Scale 17-items; YMRS: Young Mania Rating Scale; RF: Random Forest classifier; AUC: Area under the charac- teristic curve; ROC: Receiver operating characteristic; B10: Bayesian inference framework with intrinsic priors; M: Mean; SD: Standard deviation.}

\section{Acknowledgements}

The authors would like to thank the patients for participating in the studies, the nurses and PhD students involved in the studies.

\section{Authors' contributions}

MFJ, LVK and MV designed and conducted the study. MFJ, DR and JoB performed the statistical analyses. JB contributed to the design of the study and the statistical analyses. All authors have contributed to the manuscript. All authors read and approved the final manuscript.

\section{Funding}

The RADMIS trial was funded by Innovation Fund Denmark (5164-00001B9). The BIO study was funded by grants from the Mental Health Services, Capital Region of Denmark, The Danish Council for Independent Research, Medical Sciences (DFF-4183-00570), Weimans Fund, Markedmodningsfonden [the Market Development Fund, (2015-310), Gangstedfonden (A29594), Helsefonden (16-B-0063)], Innovation Fund Denmark (The Innovation Fund, Denmark, 5164-00001B), Copenhagen Center for Health Technology (CACHET), EU H2O20 ITN (EU project 722561), Augustinusfonden (16-0083), Lundbeck Foundation (R215-2015-4121). The funding organizations had no role in the design and conduct of the study; collection, management, analysis, and 
interpretation of the data; preparation, review, or approval of the manuscript; and decision to submit the manuscript for publication.

\section{Availability of data and materials}

Not applicable.

\section{Declarations}

\section{Ethics approval and consent to participate}

The RADMIS trial: the RADMIS trial was approved by the Regional Ethics Committee in The Capital Region of Denmark and the data agency, Capital Region of Copenhagen (H-16046093, RHP-2017-005, I-Suite:05365) and registered at ClinicalTrials.gov (NCT03033420). The BIO study: the study protocol was approved by the Committee on Health Research Ethics of the Capital region of Denmark (protocol No. H-7-2014-007) and the Danish Data Protection Agency, Capital Region of Copenhagen (RHP-2015-023). Both studies complied with the Declaration of Helsinki (Seoul, October 2008). All participants provided written informed consent. Data from smartphones were stored by Monsenso subject to a data management agreement between Monsenso and The Capital Region of Denmark.

\section{Consent for publication}

All authors consent for publication of the present study.

\section{Competing interests}

MFJ, DR, and JoB have no competing interests. MV has within the last 3 years been a consultant for Lundbeck, Sunovion and Janssen-Cilag. LVK has been a consultant for Lundbeck within the past 3 years. JB is a co-founder and shareholder in Monsenso.

\section{Author details}

${ }^{1}$ Copenhagen Affective Disorder Research Center (CADIC), Psychiatric Center Copenhagen, Rigshospitalet, Blegdamsvej 9, 2100 Copenhagen, Denmark. ${ }^{2}$ Department of Health Technology, Technical University of Denmark, Lyngby, Denmark. ${ }^{3}$ Department of Energy Conversion and Storage, Technical University of Denmark, Lyngby, Denmark. ${ }^{4}$ Psychiatric Centre North Zealand, Hilleroed, Denmark.

\section{Received: 13 September 2021 Accepted: 27 October 2021}

Published online: 01 December 2021

\section{References}

Anthes E. Mental health: there's an app for that. Nature. 2016;532:20-3.

Arevian AC, et al. Clinical state tracking in serious mental illness through computational analysis of speech. PLOS ONE. 2020;15:e0225695.

Baldessarini RJ, Tondo L, Baethge CJ, Lepri B, Bratti IM. Effects of treatment latency on response to maintenance treatment in manic-depressive disorders. Bipolar Disord. 2007;9:386-93.

Bardram JE, et al. Designing mobile health technology for bipolar disorder: a field trial of the monarca system. In: Proceedings of the SIGCHI conference on human factors in computing systems. France: SIGCHI; 2013. p. 2627-36.

Berry KJ, Mielke PW, Mielke HW. The Fisher-Pitman permutation test: an attractive alternative to the F test. Psychol Rep. 2002;90:495-502.

Bock JM. Medications and vocal function. Otolaryngol Clin North Am. 2019;52:693-702.

Breiman L. Random forests. Mach Learn. 2001;45:5-32.

Chawla NV, Bowyer KW, Hall LO, Kegelmeyer WP. SMOTE: synthetic minority over-sampling technique. J Artif Intell Res. 2002;16:321-57.

da Silva RDA, et al. Insight across the different mood states of bipolar disorder. Psychiatr Q. 2015;86:395-405.

da Silva RDA, Mograbi DC, Bifano J, Santana CMT, Cheniaux E. Insight in bipolar mania: evaluation of its heterogeneity and correlation with clinical symptoms. J Affect Disord. 2016;199:95-8.

Ebner-Priemer UW, et al. Digital phenotyping: towards replicable findings with comprehensive assessments and integrative models in bipolar disorders. Int J Bipolar Disord. 2020. https://doi.org/10.1186/s40345-020-00210-4.
Eyben F, Wöllmer M, Schuller B. openSMILe - the Munich versatile and fast open source audio feature extractor. Proc ACM Multimed. 2010. https:// doi.org/10.1186/s40345-020-00210-4.

Faurholt-Jepsen $\mathrm{M}$, et al. Voice analysis as an objective state marker in bipolar disorder. Transl Psychiatry. 2016;6:e856.

Faurholt-Jepsen M, et al. Reducing the rate of psychiatric Re-ADMISsions in bipolar disorder using smartphones the RADMIS trial. Acta Psychiatr Scand. 2020. https://doi.org/10.1111/acps.13274.

Fredskild MU, et al. Impact of modification to DSM-5 criterion A for hypomania/mania in newly diagnosed bipolar patients: findings from the prospective BIO study. Int J Bipolar Disord. 2021;9:14.

García S, Ramírez-Gallego S, Luengo J, Benítez JM, Herrera F. Big data preprocessing: methods and prospects. Big Data Anal. 2016;1:9.

Gideon J, Provost EM, McInnis M. Mood state prediction from speech of varying acoustic quality for individuals with bipolar disorder. Proc IEEE Int Conf Acoust Speech Signal Process. 2016;2016:2359-63.

Goodwin FK, Jamison KR. Manic-depressive illness, vol. 1996. Oxford: New Oxford University Press; 1996

Guidi A, et al. Smartphone application for the analysis of prosodic features in running speech with a focus on bipolar disorders: system performance evaluation and case study. Sensors. 2015;15:28070-87.

Hamilton M. Development of a rating scale for primary depressive illness. Br J Soc Clin Psychol. 1967;6:278-96.

Insel TR. Digital phenotyping: technology for a new science of behavior. JAMA. 2017;318:1215-6.

Karam ZN, et al. Ecologically valid long-term mood monitoring of individuals with bipolar disorder using speech. Proc Int Conf Acoust Speech Signal Process. 2014;2014:4858-62.

Kessing LV, et al. Do young adults with bipolar disorder benefit from early intervention? J Affect Disord. 2014;152-154:403-8.

Kessing LV, et al. The bipolar illness onset study: research protocol for the BIO cohort study. BMJ Open. 2017;7:e015462.

Kessing LV, et al. DSM-5 and ICD-11 criteria for bipolar disorder: Implications for the prevalence of bipolar disorder and validity of the diagnosis-a narrative review from the ECNP bipolar disorders network. Eur Neuropsychopharmacol. 2021. https://doi.org/10.1016/j.euroneuro.2021.01.097.

Lagan S, et al. Digital health developments and drawbacks: a review and analysis of top-returned apps for bipolar disorder. Int J Bipolar Disord. 2020. https://doi.org/10.1186/s40345-020-00202-4.

Leon-Novelo L, Moreno E, Casella G. Objective Bayes model selection in probit models. Stat Med. 2012;31:353-65.

Lord JR. Manic-depressive Insanity and Paranoia. By Prof. Emil Kraepelin; translated by R. Mary Barclay, M. A., M. B.; edited by George M. Robertson, M. D., F. R. C. P. Edin. Edinburgh: E. \& S. Livingstone, 1921. Demy 8vo. Pp. 280. Forty-nine illustrations, eighteen in colour. Price 12s. 6d. J Mental Sci. 1921;67:342-6.

Low DM, Bentley KH, Ghosh SS. Automated assessment of psychiatric disorders using speech: a systematic review. Laryngoscope Investig Otolaryngol. 2020;5:96-116.

Maxhuni A, et al. Classification of bipolar disorder episodes based on analysis of voice and motor activity of patients. Pervasive Mob Comput. 2016;31:50-66.

Muaremi A, Gravenhorst F, Grünerbl A, Arnrich B, Tröster G. Assessing bipolar episodes using speech cues derived from phone calls. Pervasive Comput Paradig Ment Health. 2014;2014:103-14.

Mundt JC, Vogel AP, Feltner DE, Lenderking WR. Vocal acoustic biomarkers of depression severity and treatment response. Biol Psychiatry. 2012;72:580-7.

Newman S, Mather VG. Analysis of spoken language of patients with affective disorders. AJP. 1938;94:913-42.

Olivetti E, Greiner S, Avesani P. Statistical independence for the evaluation of classifier-based diagnosis. Brain Inform. 2015;2:13-9.

Patoz M-C, et al. Patients' adherence to smartphone apps in the management of bipolar disorder: a systematic review. Int J Bipolar Disord. 2021;9:19.

Pfister T, Robinson P. Speech emotion classification and public speaking skill assessment. In: Salah AA, Gevers T, Sebe N, Vinciarelli A, editors. Human behavior understanding. Berlin: Springer; 2010. p. 151-62.

Phillips ML, Kupfer DJ. Bipolar disorder diagnosis: challenges and future directions. Lancet. 2013;381:1663-71. 
Raucher-Chéné D, Achim AM, Kaladjian A, Besche-Richard C. Verbal fluency in bipolar disorders: a systematic review and meta-analysis. J Affect Disord. 2017;207:359-66.

Schuller B, et al. The INTERSPEECH 2010 paralinguistic challenge. Japan: INTERSPEECH; 2010. p. 2794-7.

Sletved KSO, Ziersen SC, Andersen PK, Vinberg M, Kessing LV. Socio-economic functioning in patients with bipolar disorder and their unaffected siblings - results from a nation-wide population-based longitudinal study. Psychol Med. 2021. https://doi.org/10.1017/S0033291721002026.

Vanello N, et al. Speech analysis for mood state characterization in bipolar patients. Conf Proc IEEE Eng Med Biol Soc. 2012;2012:2104-7.

Vedel Kessing L, Ziersen SC, Andersen PK, Vinberg M. A nationwide population-based longitudinal study mapping psychiatric disorders during lifetime in siblings to patients with bipolar disorder. Acta Psychiatr Scand. 2021;143:284-93.

Wang K, Varma DS, Prosperi M. A systematic review of the effectiveness of mobile apps for monitoring and management of mental health symptoms or disorders. J Psychiatr Res. 2018;107:73-8.
Wickström G, Bendix T. The 'Hawthorne effect' — what did the original Hawthorne studies actually show? Scand J Work Environ Health. 2000;26:363-7.

Wing JK, et al. SCAN. Schedules for clinical assessment in neuropsychiatry. Arch Gen Psychiatry. 1990;47:589-93.

Young RC, Biggs JT, Ziegler VE, Meyer DA. A rating scale for mania: reliability, validity and sensitivity. Br J Psychiatry. 1978;133:429-35.

Zhang J, et al. Analysis on speech signal features of manic patients. J Psychiatr Res. 2018;98:59-63.

\section{Publisher's Note}

Springer Nature remains neutral with regard to jurisdictional claims in published maps and institutional affiliations.

\section{Submit your manuscript to a SpringerOpen ${ }^{\odot}$ journal and benefit from:}

- Convenient online submission

- Rigorous peer review

- Open access: articles freely available online

- High visibility within the field

- Retaining the copyright to your article

Submit your next manuscript at $\boldsymbol{\nabla}$ springeropen.com 